\title{
Cancer detection rate of prebiopsy MRI with subsequent systematic and targeted biopsy are superior to non-targeting systematic biopsy without MRI in biopsy naïve patients: a retrospective cohort study
}

Satoshi Washino ${ }^{1,3^{*}}$, Shigeru Kobayashi ${ }^{2}$, Tomohisa Okochi ${ }^{4}$, Tomohiro Kameda ${ }^{1}$, Tsuzumi Konoshi ${ }^{3}$, Tomoaki Miyagawa ${ }^{3}$, Tatsuya Takayama ${ }^{1}$ and Tatsuo Morita ${ }^{1}$

\begin{abstract}
Background: To determine whether prebiopsy multiparametric magnetic resonance imaging (mpMRI) with subsequent systematic plus targeted biopsies for suspicious lesions improve prostate cancer detection compared with standard non-targeting systematic biopsies without mpMRI in biopsy-naïve patients.

Methods: Patients who underwent their first prostate biopsy due to suspicion of prostate cancer were analyzed retrospectively to compare the biopsy outcomes between patients who received prebiopsy mpMRI (215 patients) and those who did not (281 patients). mpMRI was performed to determine pre-biopsy likelihood of the presence of prostate cancer using a three-point scale ( $1=$ low level of suspicion, $2=$ equivocal, and $3=$ high level of suspicion). Systematic biopsies were performed in both groups. Targeted biopsies were added for a high level of suspicious lesions on mpMRI. All biopsies were performed by transperineal biopsy technique. After biopsy, Prostate Imaging Reporting and Data System ver. 2 (PIRADS-2) scoring was performed to describe the mpMRI findings and predictive value of PIRADS-2 was evaluated.

Results: The detection rate of total and clinically significant prostate cancer was significantly higher in patients who received prebiopsy mpMRI than in those who did not (55.3 and $46.0 \%$ vs. 42.0 and 35.2\%, respectively; $p=0.004$ and $p=0.016$ ). The clinically insignificant prostate cancer detection rate was similar between the two groups (9.3\% vs. $6.8 \% ; p=0.32$ ). Of 86 patients who underwent systematic plus targeted biopsy in the MRI cohort and were diagnosed with prostate cancer, seven patients were detected by addition of targeted biopsy whereas 29 patients were missed by targeted biopsy but detected by systematic biopsy. There was a correlation between the PIRADS-2 and prostate cancer detection rate, and a receiver-operator curve analysis yielded an area under the curve of $0.801(p<0.0001)$.
\end{abstract}

Conclusions: Prebiopsy mpMRI with subsequent systematic plus targeted biopsies for suspicious lesions can yield a higher cancer detection rate than non-targeting systematic biopsies. PIRADS-2 scoring is useful for predicting the biopsy outcome.

Keywords: Magnetic resonance imaging, Biopsy, Target, Random, Prostate cancer

\footnotetext{
* Correspondence: suwajiisan@jichi.ac.jp

'Department of Urology, Jichi Medical University, 3311-1 Yakushiji,

Shimotsuke, Tochigi 329-0498, Japan

${ }^{3}$ Department of Urology, Jichi Medical University Saitama Medical Center,

1-847, Amanuma-cho, Omiya-ku, Saitama 330-8503, Japan

Full list of author information is available at the end of the article
}

(c) The Author(s). 2018 Open Access This article is distributed under the terms of the Creative Commons Attribution 4.0 International License (http://creativecommons.org/licenses/by/4.0/), which permits unrestricted use, distribution, and reproduction in any medium, provided you give appropriate credit to the original author(s) and the source, provide a link to the Creative Commons license, and indicate if changes were made. The Creative Commons Public Domain Dedication waiver (http://creativecommons.org/publicdomain/zero/1.0/) applies to the data made available in this article, unless otherwise stated. 


\section{Background}

Prostate cancer ( $\mathrm{PCa})$ is the most common male malignancy and the second most common cause of male cancer-related death [1]. It is usually diagnosed based on systematic transrectal ultrasound (TRUS)-guided random biopsies of the prostate gland. However, a significant number of transrectal biopsies are negative for cancer, yielding inaccurate results [2-4]. The cancer detection rate with a standard TRUS-guided prostate biopsy is only $20 \sim 40 \%$ [5]. Furthermore, TRUS-guided transrectal biopsies have a limited ability to sample the anterior prostate [6].

The ideal prostate biopsy goal would be to identify clinically significant $\mathrm{PCa}$ and minimize the detection of indolent disease. The growing availability of prostate multiparametric magnetic resonance imaging (mpMRI), novel functional imaging modalities, and increased standardization have created an opportunity for the detection, localization, and staging of $\mathrm{PCa}$ [7]. Screening patients using mpMRI may avoid the morbidity associated with a biopsy if no lesions are seen [8]. In addition, targeted biopsies should identify more clinically significant PCa than non-targeted TRUS-guided biopsies [9]. High PCa detection rates have been reported using magnetic resonance imaging (MRI)-targeted biopsies, both in patients with prior negative TRUS biopsies and in biopsy-naïve patients [9-13]. However, it is not clear whether prebiopsy MRI with a subsequent targeted biopsy is superior to the traditional systematic non-targeted TRUS biopsy in biopsy-naive patients [14-16]. Therefore, this study examined whether prebiopsy mpMRI with the subsequent addition of targeted biopsies for suspicious lesions could improve the $\mathrm{PCa}$ detection rate in biopsy-naïve patients. These results were compared with those of a standard cohort of patients who underwent systematic non-targeted TRUS biopsies without MRI.

\section{Methods \\ Patients}

This retrospective observational study was approved by the local Institutional Review Board. The eligibility criteria were as follows: patients with a prostate-specific antigen (PSA) level $<15 \mathrm{ng} / \mathrm{mL}$ who underwent their first prostate biopsy for suspected PCa at Jichi Medical University or Jichi Medical University Saitama Medical Center between January 2010 and April 2014. Patients who underwent mpMRI before their prostate biopsy were assigned to the MRI cohort, whereas those who did not undergo MRI were assigned to the non-MRI cohort. Physicians decided who was to undergo MRI as their beliefs and patients' preferences after discussion with patients. A total of 557 patients were eligible: 383 at Jichi Medical University and 174 at Jichi Medical University Saitama Medical Center. Sixty-one patients were excluded based on the following criteria: interval from
mpMRI to biopsy $>6$ months ( 25 patients), $<12$ biopsy cores (17 patients), MRI performed in another hospital (11 patients), the use of $5 \alpha$-reductase inhibitors or anti-androgen therapy at the time of biopsy ( 6 patients), previous prostate surgical intervention (1 patient), and a blurred MRI scan (1 patient). Data from 496 patients were analyzed, and 215 and 281 patients were assigned to the MRI and non-MRI cohorts, respectively.

\section{Imaging}

All patients in the MRI cohort underwent mpMRI, which was performed using a 1.5-Tesla (Excelart Vantage, Toshiba Medical Systems, Otawara, Japan; MAGNETOM Symphony Advanced, Siemens, Munich, Germany; MAGNEOM Avanto, Siemens; or Achieva, Philips, Amsetrdam, Netherlands) or 3-Tesla (Vantage Titan 3 T, Toshiba Medical Systems; or MAGNETOM Skyra, Siemens) machine with a 16-channel phased-array body coil. The protocol included T2-weighted imaging, diffusion-weighted imaging, and dynamic contrast-enhanced imaging. Radiologists evaluated the mpMRI results and determined the locations of suspicious lesions. The likelihood of the presence of $\mathrm{PCa}$ was determined using a three-point scale $(1=$ low level of suspicion, $2=$ equivocal, and $3=$ high level of suspicion) because the standardized Prostate Imaging Reporting and Data System (PIRADS) criteria were not used to evaluate the images when the biopsies were performed. However, one very experienced genitourinary radiologist (T.O. or S.K.) at each institute blinded to the biopsy and the first MRI evaluation before biopsy reviewed and scored each suspicious lesion in the mpMRI image from 1 to 5 points according to the PIRADS criteria (ver. 2.0; PIRADS-2) [17, 18]. PI-RADS is both quantitative and qualitative, but only qualitative scoring was used in this study. The highest overall PIRADS-2 score of each mpMRI scan was used.

\section{Biopsy protocol}

All biopsies were performed using a transperineal approach with an 18-gauge needle biopsy gun under general or spinal anesthesia. In the non-MRI cohort, 12 to 14 cores were biopsied during non-targeted systematic TRUS-guided transperineal biopsies. In the MRI cohort, 12 to 14 cores were also biopsied during systematic TRUS-guided transperineal biopsies. However, in patients who had suspicious lesions on mpMRI, each lesion was targeted in one of the systematic biopsies and, typically, two cores of targeted biopsies were added for each lesion (Fig. 1). The targeted biopsies were performed using the cognitive registration technique described previously [19], with a minor modification: we used an ultrasound-guided freehand biopsy instead of a transperineal template. 


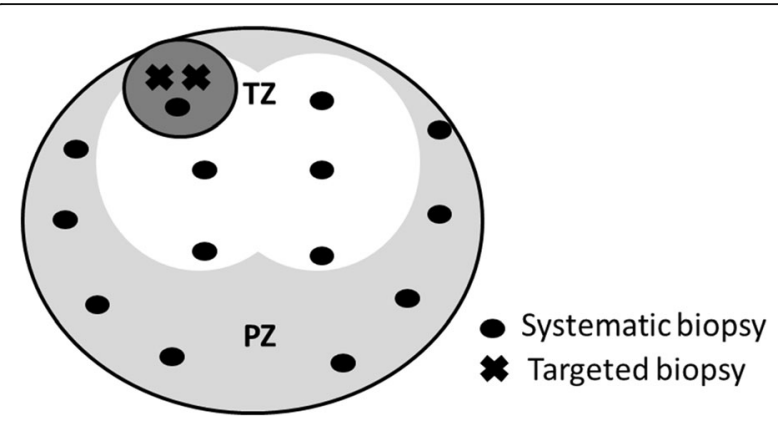

Fig. 1 Biopsy strategy. In the MRI cohort, 12 to 14 cores were biopsied. In patients who had suspicious lesions on mpMRl, each suspicious lesion could be targeted as one of systematic biopsy at the nearest point and further typically two targeted biopsies were added for each lesion. White, light gray, and dark gray areas indicate transitional zone, peripheral zone of axial view, and index lesion on mpMRl, respectively. Black dot indicates systematic biopsy cores and $\mathrm{x}$ indicates targeted biopsy cores. TZ; transitional zone, PZ: peripheral zone

\section{Clinically significant cancer}

Clinically significant PCa was defined as a Gleason score (GS) $\geq 3+4$ or a maximum cancer core length $\geq 4 \mathrm{~mm}$; all other lesions were deemed as clinically insignificant PCa. This threshold has been validated to predict lesions with tumor volumes $\geq 2 \mathrm{~mL}$ [19].

\section{Study endpoints}

Patients' characteristics and cancer detection rate were compared between the MRI and non-MRI cohort. The endpoint of this study was the detection rate of all $\mathrm{PCa}$ and clinically significant PCa.

\section{Statistical analysis}

The data were analyzed using the Mann-Whitney $U$ test or Fisher's exact test. Univariate and multivariate analyses were performed using logistic regression analysis to determine significant predictors of PCa. A $p$-value $\leq 0.05$ was considered significant. The statistical analyses were performed using GraphPad Prism (ver. 5.0; GraphPad, La Jolla, CA, USA) and SPSS for Windows software (ver. 19.0; SPSS Inc., Chicago, IL, USA).

\section{Results}

Patient characteristics, MRI images, and biopsy strategy There were no significant differences in patient characteristics between the two cohorts, except for prostate volume, which was significantly smaller in the MRI cohort than in the non-MRI cohort (median $=27.7$ vs. $32.0 \mathrm{~cm}^{3}, p=0.0002$; Table 1). Examples of 1.5 - and 3-Tesla MRI images are shown in Fig. 2. The 3-Tesla MRI seems to show the suspicious lesion clearly, compared to 1.5-Tesla MRI. Systematic biopsies were

Table 1 Patient characteristics and biopsy outcomes

\begin{tabular}{|c|c|c|c|c|c|}
\hline & \multicolumn{2}{|l|}{$\begin{array}{l}\text { MRI (+) } \\
n=215\end{array}$} & \multicolumn{2}{|l|}{$\begin{array}{l}\text { MRI (-) } \\
n=281\end{array}$} & \multirow[t]{2}{*}{$p$ Value } \\
\hline & Median & IQR & Median & IQR & \\
\hline Age & 68 & $(62-72)$ & 68 & $(63-72)$ & 0.21 \\
\hline PSA (ng/mL) & 6.4 & $(5.2-8.8)$ & 6.7 & $(5.5-9.4)$ & 0.25 \\
\hline Prostate volume $\left(\mathrm{cm}^{3}\right)$ & 27.7 & $(21.0-36.0)$ & 32.0 & $(23.0-45.8)$ & 0.0002 \\
\hline PSA density $\left(\mathrm{ng} / \mathrm{mL} / \mathrm{cm}^{3}\right)$ & 0.22 & $(0.16-0.34)$ & 0.23 & $(0.16-0.34)$ & 0.79 \\
\hline DRE positive, n (\%) & 44 & $(20.5)$ & 41 & $(14.6)$ & 0.09 \\
\hline TRUS positive, n (\%) & 32 & $(14.9)$ & 29 & $(10.3)$ & 0.13 \\
\hline \multicolumn{6}{|l|}{ MRI type, n (\%) } \\
\hline 1.5 Tesla & 161 & $(74.9 \%)$ & $(-)$ & & \\
\hline 3 Tesla & 54 & $(25.1 \%)$ & $(-)$ & & \\
\hline Prostate cancer, n (\%) & 119 & $(55.3 \%)$ & 118 & $(42.0 \%)$ & 0.004 \\
\hline \multicolumn{6}{|l|}{ Gleason sum, n (\%) } \\
\hline $3+3$ & 34 & $(15.8 \%)$ & 42 & $(14.9 \%)$ & 0.80 \\
\hline $3+4$ & 43 & $(20.0 \%)$ & 40 & $(14.5 \%)$ & 0.08 \\
\hline $4+3$ & 16 & $(7.4 \%)$ & 11 & $(3.9 \%)$ & 0.11 \\
\hline 8 or more & 26 & $(12.1 \%)$ & 25 & $(8.9 \%)$ & 0.30 \\
\hline \multicolumn{6}{|l|}{ Clinical significance, n (\%) } \\
\hline Insignificant cancer & 20 & $(9.3 \%)$ & 19 & $(6.8 \%)$ & 0.32 \\
\hline Significant cancer & 99 & $(46.0 \%)$ & 99 & $(35.2 \%)$ & 0.016 \\
\hline Cancer positive cores & 3 & $(1.25-5)$ & 2 & $(1-4)$ & 0.28 \\
\hline
\end{tabular}


performed in all patients and the median number of cores collected was 12 [interquartile range $(\mathrm{IQR})=12-14$ ] in both cohorts. Targeted biopsies for suspicious lesions on mpMRI were performed in 129 patients $(60.0 \%)$ in the MRI cohort; 345 cores were targeted for 145 suspicious lesions; the median targeted core number per prostate was two (IQR $=2-4)$.

\section{Cancer detection rate}

The cancer detection rate was significantly higher in the MRI cohort than in the non-MRI cohort $(55.3 \%$ vs. $42.0 \%, p=0.004$; Table 1 ). When cancer grade was compared between the two cohorts, there was no significant difference in the detection rate of low-grade cancer (GS $3+3 ; 15.8 \%$ in the MRI cohort vs. $14.9 \%$ in the non-MRI cohort, $p=0.80$ ). However, detection of intermediate- or high-grade cancer $(G S \geq 3+4)$ was significantly higher in the MRI cohort compared with the non-MRI cohort (39.5\% vs. $27.0 \%$, respectively, $p=0.004)$. The clinically significant $\mathrm{PCa}$ detection rate was also higher in the MRI cohort than in the non-MRI cohort $(46.0 \%$ vs. $35.2 \%$, respectively, $p=0.016$ ).

\section{Systematic and targeted cores in each cohort}

Table 2 summarizes the outcomes of the systematic and targeted cores in each cohort. The rate of cancer-positive cores and cores including a Gleason pattern of 4 or more was significantly higher in targeted biopsies than in systematic biopsies in the MRI and non-MRI cohorts, respectively (31.3 and $23.1 \%$ vs. 12.3 and $8.4 \%$, and 10.0 and $5.9 \%$, all $p<0.0001$ ) (Table 2). The cancer-positive core rate for systematic biopsies in the MRI cohort was also significantly higher than that in the non-MRI cohort $(12.3 \%$ vs. $10.0 \%, p=0.004)$. The percentage of core length involved by cancer in targeted cores was also significantly higher than that in systematic biopsies, in both the MRI and non-MRI cohorts $[$ median $(\mathrm{IQR})=50 \%(20-70)$ vs. $30 \%(10-60)$ and $20 \%(10-50), p=0.0011$ and $p<0.0001]$.

\section{Addition of targeted biopsies to systematic biopsies}

Of the 129 patients who underwent systematic and targeted biopsies in the MRI cohort, 86 had PCa. We performed a subgroup analysis on these patients to assess the performance of the targeted biopsies (Fig. 3). Seven and four patients were diagnosed with $\mathrm{PCa}$ and upgraded to an intermediate and high grade, respectively, by the addition of targeted biopsies. The index lesions in seven patients missed by systematic biopsies but detected by targeted biopsies were in the anterior transitional zone $(n=4)$, anterior stroma $(n=1)$, anterior peripheral zone $(n=1)$, and posterolateral peripheral zone $(n=1)$. Twenty-nine patients were missed by targeted biopsies but detected by systematic biopsies.

\section{Factors predicting prostate cancer detection}

Univariate and multivariate analyses were performed to identify predictors of PCa detection (Table 3). Multivariate logistic regression analysis revealed that PSA level, prostate volume, performing prebiopsy mpMRI, and digital rectal examination findings were independent predictors of $\mathrm{PCa}$ detection.

\section{Cancer detection rate according to PIRADS-2 score}

The detection rate of all $\mathrm{PCa}$ and clinically significant PCa, stratified according to PIRADS-2 scores of 1, 2, 3, 4, and 5 in the MRI cohort, was 31.8 and $18.2 \%, 9.1$ and 9.1\%, 14.5 and $10.9 \%, 78.3$ and $66.0 \%$, and 83.3 and $80.0 \%$, respectively (Table 4). The detection rate of clinically significant cancer was significantly higher in patients with

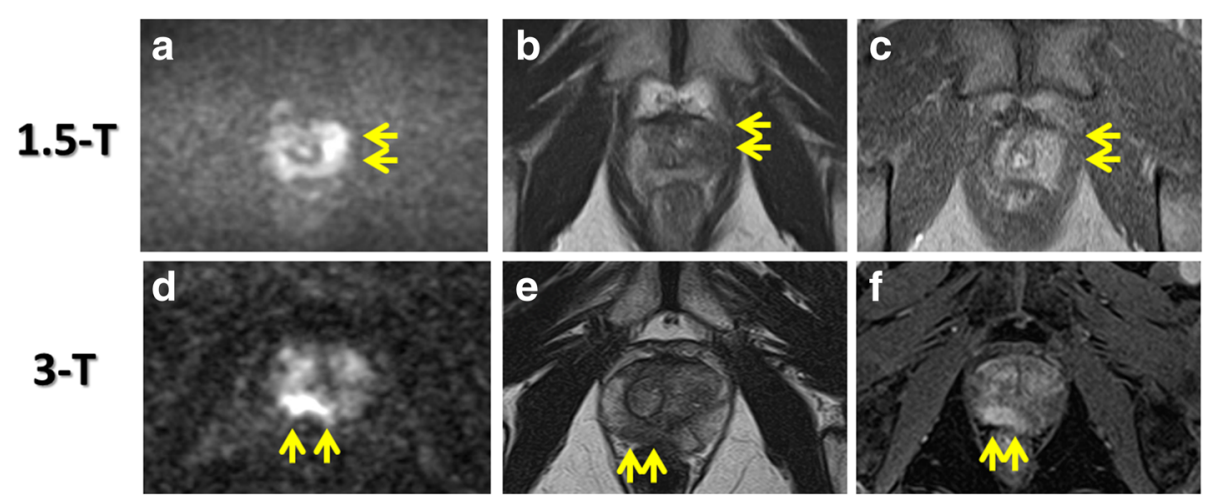

Fig. 2 MRI images. 1.5-Tesla MRI images obtained from a patient with a PSA level of $3.86 \mathrm{ng} / \mathrm{mL}$ (A - C). Diffusion weighted image (DWI) with a b-value $1500 \mathrm{~s} / \mathrm{mm}^{2}$ (A) showed a high intensity area in the left peripheral zone (Arrow). T2 weighted image (T2Wl: B) and dynamic contrast enhancement image (DCEl: C) showed a low intensity area and an enhancement, respectively, in the same lesion, which was considered to be a high level of suspicious. 3-Tesla MRI images obtained from a patient with a PSA level of $3.57 \mathrm{ng} / \mathrm{mL}$ (D - F). DWI with a b-value $2000 \mathrm{~s} / \mathrm{mm}^{2}$ (D) showed a high intensity area in the right peripheral zone (Arrow). T2WI (E) and dynamic DCEI (F) showed a low intensity area and an enhancement, respectively, in the same lesion, which was considered to be a high level of suspicious 
Table 2 Systematic and targeted cores in each cohort

\begin{tabular}{|c|c|c|c|c|c|c|c|c|c|}
\hline \multirow[b]{3}{*}{ Cores of cancer, n (\%) } & \multicolumn{4}{|c|}{ MRI (+) } & \multirow{2}{*}{\multicolumn{2}{|c|}{$\begin{array}{l}\text { MRI }(-) \\
\text { Systematic cores } \\
n=3542(C)\end{array}$}} & \multicolumn{3}{|l|}{$p$ Value } \\
\hline & \multicolumn{2}{|c|}{$\begin{array}{l}\text { Systematic cores } \\
n=2696 \text { (A) }\end{array}$} & \multicolumn{2}{|c|}{$\begin{array}{l}\text { Targeted cores } \\
n=345(\mathrm{~B})\end{array}$} & & & \multirow{2}{*}{$\begin{array}{l}A \text { vs B } \\
<0.0001\end{array}$} & \multirow{2}{*}{$\begin{array}{l}\text { A vs } C \\
0.004\end{array}$} & \multirow{2}{*}{$\begin{array}{l}\text { B vs C } \\
<0.0001\end{array}$} \\
\hline & 333 & $(12.3)$ & 108 & $(31.3)$ & 355 & $(10.0)$ & & & \\
\hline Cores including GP 4 or more, n (\%) & 229 & $(8.4)$ & 80 & $(23.1)$ & 210 & $(5.9)$ & $<0.0001$ & 0.0001 & $<0.0001$ \\
\hline $\begin{array}{l}\text { Median percentage of core length } \\
\text { involved by cancer (IQR) }\end{array}$ & 30 & $(10-60)$ & 50 & $(20-70)$ & 20 & $(10-50)$ & 0.0011 & 0.10 & $<0.0001$ \\
\hline
\end{tabular}

a PIRADS-2 score of 4 or 5 compared with those with a PIRADS-2 score of $1-3$ (69.3\% vs. $12.5 \%$; $p<0.0001)$. By contrast, there was no significant difference in the detection rate of clinically insignificant $\mathrm{PCa}$ between groups $(10.2 \%$ vs. $5.7 \% ; p=0.32)$. When a PIRADS- 2 score of 4 or 5 was considered positive, the sensitivity, specificity, positive predictive value (PPV), and negative predictive value (NPV) for PCa detection were 0.87 [95\% confidence intervals (CI), 0.79-0.92], 0.75 (95\% CI, 0.65-0.83), 0.81 (95\% CI, 0.73-0.88), and 0.82 (95\% CI, 0.72-0.89), respectively. Receiver operating characteristic (ROC) curve analysis for predicting $\mathrm{PCa}$ detection using the PIRADS-2 score revealed an area under the curve (AUC) of 0.801 (95\% CI, 0.738-0.864), which was superior to AUC of 0.738 (95\% $\mathrm{CI}, 0.670-0.806)$ in the three-point scale at the first radiological evaluation before biopsy.

\section{Discussion}

In this study, the detection rate of clinically significant PCa was significantly higher in the MRI cohort than the non-MRI cohort, whereas the clinically insignificant PCa detection rate was similar in both groups (Table 1). Performing MRI was an independent predictor of $\mathrm{PCa}$ detection (Table 3 ). These results suggest that prebiopsy MRI has the potential to improve biopsy outcomes in biopsy-naïve patients. We believe that a prebiopsy MRI has at least two advantages: patients can be selected more efficiently and a targeted biopsy of the index lesion can be added.
Traditionally, the decision regarding whether a prostate biopsy should be performed has been based mainly on the PSA, digital rectal examination findings, and age, which leads to inaccurate results. MRI can provide more precise information about the likelihood of the presence of PCa and prostate volume before biopsy. The prostate volume is negatively associated with the cancer detection rate, as shown in this (Table 3) and previous studies [20] and prostate volume was significantly smaller in the MRI cohort than the non-MRI cohort in this study (Table 1). These suggest that prostate biopsies might not be recommended in patients with a large prostate volume and/or normal MRI. Patient selection may partially explain the higher cancer detection rate in the MRI cohort. The combination of MRI findings and other biomarkers may better determine which patients should undergo prostate biopsy. Recently, we reported that the combination of PIRADS-2 score and PSA density was useful for decision-making before a prostate biopsy [21]. In that study, no patients with a PIRADS-2 score of $\leq 3$ and PSA density of $<0.15 \mathrm{ng} / \mathrm{mL} / \mathrm{cm}^{3}$ were diagnosed with clinically significant PCa. In the present study, 27 patients had a PIRADS-2 score of $\leq 3$ and PSA density of $<0.15 \mathrm{ng} / \mathrm{mL} / \mathrm{cm}^{3}$. Of these, no patients were diagnosed with clinically significant PCa (data not shown).

In this study, targeted biopsies based on MRI detected PCa more efficiently than systematic biopsies (Table 2). Furthermore, some patients in the MRI cohort were diagnosed with $\mathrm{PCa}$ or upgraded by the addition of targeted biopsies (Fig. 3). In particular, targeted biopsy

\begin{tabular}{|c|c|c|c|c|c|c|}
\hline & & \multicolumn{4}{|c|}{ Targeted } & \multirow[b]{2}{*}{ Total } \\
\hline & & No cancer & GS $3+3$ & GS 7 & GS 8 or more & \\
\hline \multirow{4}{*}{ Systematic } & No cancer & 0 & 4 & 3 & 0 & 7 \\
\hline & GS $3+3$ & 8 & 11 & 2 & 0 & 21 \\
\hline & GS 7 & 17 & 2 & 21 & 2 & 42 \\
\hline & GS 8 or more & 4 & 0 & 1 & 11 & 16 \\
\hline & Total & 29 & 17 & 27 & 13 & 86 \\
\hline
\end{tabular}

Fig. 3 Cross-tabulation of histology (Gleason score) of targeted and systematic biopsy among patients who received both biopsies and had prostate cancer. Seven patients were diagnosed with prostate cancer by addition of targeted biopsies (dark gray box). Additionally, four patients were upgraded to intermediate or high grade by addition of targeted biopsies (light gray box). GS, Gleason score 
Table 3 Univariate and multivariate analysis for prostate cancer detection in all patients

\begin{tabular}{|c|c|c|c|c|c|c|c|c|}
\hline & & \multirow[b]{2}{*}{$\mathrm{N}$} & \multicolumn{3}{|c|}{ Univariate analysis } & \multicolumn{3}{|c|}{ Multivariate analysis } \\
\hline & & & $\mathrm{HR}$ & $95 \% \mathrm{Cl}$ & $p$-value & $\mathrm{HR}$ & $95 \% \mathrm{Cl}$ & $p$-value \\
\hline \multirow[t]{3}{*}{ Age } & $\leq 65$ & 189 & - & - & - & $(-)$ & $(-)$ & 0.101 \\
\hline & $66-70$ & 147 & 1.166 & $0.755-1.801$ & 0.488 & & & \\
\hline & $>70$ & 160 & 1.836 & $1.119-2.812$ & 0.005 & & & \\
\hline \multirow[t]{4}{*}{ PSA } & $\leq 5.0$ & 89 & - & - & - & - & - & - \\
\hline & $5.01-7.50$ & 219 & 1.194 & $0.721-1.978$ & 0.491 & 1.346 & $0.767-2.363$ & 0.300 \\
\hline & $7.51-10$ & 97 & 1.949 & $1.085-3.499$ & 0.026 & 2.620 & $1.342-5.115$ & 0.005 \\
\hline & $>10.0$ & 91 & 2.712 & $1.484-4.955$ & 0.001 & 3.947 & $1.995-7.806$ & $<0.001$ \\
\hline \multirow[t]{3}{*}{ Prostate volume } & $\leq 25.0$ & 176 & - & - & - & - & - & - \\
\hline & $25.1-35.0$ & 147 & 0.361 & $0.228-0.571$ & $<0.001$ & 0.336 & $0.205-0.551$ & $<0.001$ \\
\hline & $>35.0$ & 173 & 0.135 & $0.084-0.217$ & $<0.001$ & 0.112 & $0.073-0.204$ & $<0.001$ \\
\hline \multirow[t]{2}{*}{ MRI } & No & 281 & - & - & - & - & - & - \\
\hline & Yes & 215 & 1.712 & $1.197-2.450$ & 0.003 & 1.749 & $1.160-2.636$ & 0.008 \\
\hline \multirow[t]{2}{*}{ DRE } & Negative & 411 & - & - & - & - & - & - \\
\hline & Positive & 85 & 4.193 & $2.467-7.126$ & $<0.001$ & 3.068 & $1.704-5.526$ & $<0.001$ \\
\hline \multirow[t]{2}{*}{ TRUS } & Negative & 435 & - & - & - & $(-)$ & $(-)$ & 0.154 \\
\hline & Positive & 61 & 2.500 & $1.418-4.407$ & 0.002 & & & \\
\hline
\end{tabular}

was beneficial for the diagnosis of anterior cancer because most of the tumors detected by targeted biopsies, but missed by systematic biopsies, were located in the anterior zone. Collectively, the addition of a targeted biopsy might improve the biopsy outcome. However, the effectiveness of targeted biopsy in this study may have been underestimated because the urologist was not blinded to the suspicious lesions on MRI, so that these could be targeted in systematic biopsies. This suggestion is supported by the fact that the cancer-positive core rate in systematic biopsies in the MRI cohort was significantly higher than that in the non-MRI cohort (Table 2). However, performing the study with the urologists blinded to the MRI during the systematic biopsies would be preferred to evaluate the effectiveness of systematic and targeted biopsies accurately. The cancer detection rate of $55.3 \%$ in the MRI cohort in this study was somewhat lower than that reported in previous series that performed targeted biopsies with or without systematic biopsies (56-64\%) [10, 13, 22, 23]. Furthermore, one third of the tumors were missed by targeted biopsies, but detected by systematic biopsies (Fig. 3). Three techniques have been reported for targeted biopsies: MRI-guided in-bore biopsy; MRI-TRUS fusion-guided biopsy; and cognitive registration. Although few direct comparisons have been performed, MRI-guided in-bore and MRI-TRUS fusion-guided biopsies likely yield a higher detection rate of clinically significant PCa compared with cognitive registration [13, 14, 22-24]. The cognitive registration technique was used in this study, which may have caused the somewhat low PCa detection rate. However, MRI-guided in-bore and MRI-TRUS fusion-guided biopsies have limited availability and are complex and costly to introduce and/or perform; therefore, they are not feasible for routine use so far. These suggest that targeted biopsies using cognitive registration are more practical and may improve the performance of prostate biopsy.

This study found a correlation between the PIRADS-2 score and $\mathrm{PCa}$ detection rate, especially in clinically significant PCa. ROC analysis revealed an AUC of 0.801 which was superior to that of 0.738 in the three-point scale; the sensitivity, specificity, PPV, and NPV were $0.87,0.75,0.81$, and 0.82 , respectively. A meta-analysis

Table 4 Cancer detection rate according to PI-RADS v2 score

\begin{tabular}{|c|c|c|c|c|c|}
\hline & \multicolumn{5}{|c|}{ PI-RADS v2 score } \\
\hline & $\begin{array}{l}1 \\
n=22\end{array}$ & $\begin{array}{l}2 \\
n=11\end{array}$ & $\begin{array}{l}3 \\
n=55\end{array}$ & $\begin{array}{l}4 \\
n=97\end{array}$ & $\begin{array}{l}5 \\
n=30\end{array}$ \\
\hline Insignificant PCa, n (\%) & $3(13.6 \%)$ & $0(0 \%)$ & $2(3.6 \%)$ & $12(12.4 \%)$ & $1(3.3 \%)$ \\
\hline Significant PCa, n (\%) & $4(18.2 \%)$ & $1(9.1 \%)$ & $6(10.9 \%)$ & $64(66.0 \%)$ & $24(80.0 \%)$ \\
\hline Total PCa, n (\%) & $7(31.8 \%)$ & $1(9.1 \%)$ & $8(14.5 \%)$ & $76(78.3 \%)$ & $25(83.3 \%)$ \\
\hline
\end{tabular}


assessing the performance of mpMRI for detecting PCa found a specificity of 0.88 (95\% CI, 0.82-0.92), sensitivity of 0.74 (95\% CI, 0.66-0.81), and NPV of 0.64-0.94, which is consistent with our results. Recently, PIRADS was annotated, revised, and published as a second version, PIRADS-2, to define standards of high-quality clinical service for mpMRI, including image creation and reporting [17, 18]. Kuru et al. performed ROC analysis for PCa detection using the PIRADS score and found an excellent specificity of $0.90-0.98$ [25]. Grey et al. [26] reported that a ROC analysis of clinically significant $\mathrm{PCa}$ yielded an AUC of $0.88-0.89$ and sensitivity, specificity, PPV, and NPV of 0.95-0.97, 0.6, 0.58-0.61, and $0.97-0.98$, respectively. The difference in the ability to predict biopsy outcome among studies may be due to differences in the MRI machines and protocols used, as well as to variations in the PIRADS scoring, biopsy protocols, and patient characteristics.

Two recent randomized controlled trials (RCTs) compared the outcomes between prebiopsy MRI with the addition of a targeted biopsy and a conventional TRUS-guided random biopsy $[15,16]$. One used a MRI-TRUS fusion targeted biopsy, while the other used the cognitive registration technique, similar to our study. Both found that the PCa detection rate was similar between the MRI and control groups, which is not consistent with our results. Selection bias in our retrospective study could have been the major reason for the difference versus the two RCTs, and patient selection after MRI may have been one of the major causes of the higher cancer detection rate in our MRI cohort. There were also differences in the study protocols, sample sizes, and biopsy protocols among studies. Furthermore, the PCa detection rate of $54-57 \%$ for the conventional TRUS-guided biopsy of the two RCTs was higher than that in our study, and another reported study [5].

This study had some limitations. First, the analysis was retrospective and patient selection bias may have been present, as described above. However, it appears based on the statistics that the two cohorts were very similar other than prostate size. Second, PIRADS scoring was not performed at the same time as the biopsy. Third, some patients underwent 1.5-Tesla MRI, whereas others underwent 3-Tesla MRI, reflecting technological changes. However, the PCa detection rate did not differ significantly between 1.5 -Tesla and 3-Tesla MRI (data not shown). Fourth, there is no widely accepted definition of clinically significant PCa. Fifth, the promise of targeted biopsies is to reduce the number of total biopsies. However, our study suggests that systematic biopsies should not be omitted when cognitive fusion transperineal biopsies are performed because one third of the tumors were missed by targeted biopsies but detected by systematic biopsies (Fig. 3). Sixth, several radiologists were involved in reporting the MRIs and several urologists performed biopsies. However, this could be deemed an advantage, since clinical effectiveness of prebiopsy mpMRI was demonstrated despite this heterogeneity. Finally, only one radiologist was involved in the MRI review of the PIRADS-2 scoring in each institution; therefore, inter-observer reliability could not be assessed.

\section{Conclusions}

Prebiopsy mpMRI with subsequent systematic plus targeted biopsies could yield a more clinically significant $\mathrm{PCa}$ detection rate than a non-targeted TRUS-guided biopsy in biopsy-naïve patients. PIRADS-2 scoring is useful for predicting biopsy outcome. However, large prospective studies are needed to confirm our results.

\section{Abbreviations}

AUC: Area under the curve; Cl: Confidence intervals; GS: Gleason score; IQR: Interquartile range; mpMRI: multiparametric magnetic resonance imaging; MRI: Magnetic resonance imaging; NPV: Negative predictive values; PCa: Prostate cancer; PIRADS: Prostate imaging reporting and data system; PIRADS-2: Prostate imaging reporting and data system version 2.0; PPV: Positive predictive values; ROC: Receiver operating characteristic; TRUS: Transrectal ultrasound

\section{Funding}

There was no financial or material support for this article.

Availability of data and materials

The datasets analyzed during the current study is available from the corresponding author on reasonable request.

\section{Authors' contributions}

SW contributed to the study design, data collection, interpretation and manuscript writing. SK, TO, T Kameda, and T Konishi contributed to data collection and interpretation. T Miyagawa, TT, and T Morita contributed to data analysis and manuscript writing. All authors read and approved the final manuscript.

Ethics approval and consent to participate

The study protocol was reviewed and approved by the Institutional Review Board at Jichi Medical University or Jichi Medical University Saitama Medical Center (Rin15-29 and RinA14-096) and obtaining additional informed consent from patients was not required by the ethics committee for this retrospective study.

\section{Competing interests}

The authors declare that they have no competing interests.

\section{Publisher's Note}

Springer Nature remains neutral with regard to jurisdictional claims in published maps and institutional affiliations.

\section{Author details}

${ }^{1}$ Department of Urology, Jichi Medical University, 3311-1 Yakushiji, Shimotsuke, Tochigi 329-0498, Japan. ²Department of Radiology, Jichi Medical University, 3311-1 Yakushiji, Shimotsuke, Tochigi 329-0498, Japan. ${ }^{3}$ Department of Urology, Jichi Medical University Saitama Medical Center, 1-847, Amanuma-cho, Omiya-ku, Saitama 330-8503, Japan. ${ }^{4}$ Department of Radiology, Jichi Medical University Saitama Medical Center, 1-847, Amanuma-cho, Omiya-ku, Saitama 330-8503, Japan. 
Received: 11 February 2017 Accepted: 10 May 2018

Published online: 30 May 2018

\section{References}

1. Center MM, Jemal A, Lortet-Tieulent J, Ward E, Ferlay J, Brawley O, Bray F. International Variation in Prostate Cancer Incidence and Mortality Rates. European Urology. 2012;61(6):1079-92.

2. Sinnott M, Falzarano SM, Hernandez AV, Jones JS, Klein EA, Zhou M, Magi-Galluzzi C. Discrepancy in prostate cancer localization between biopsy and prostatectomy specimens in patients with unilateral positive biopsy: implications for focal therapy. Prostate. 2012;72(11):1179-86.

3. Han M, Chang D, Kim C, Lee BJ, Zuo Y, Kim H-J, Petrisor D, Trock B, Partin AW, Rodriguez R, et al. Geometric evaluation of systematic transrectal ultrasound guided prostate biopsy. J Urol. 2012;188(6):2404-9.

4. de Rooij M, Hamoen EHJ, Futterer JJ, Barentsz JO, Rovers MM. Accuracy of multiparametric MRI for prostate Cancer detection: a meta-analysis. Am J Roentgenol. 2014;202(2):343-51.

5. Trabulsi EJ, Halpern EJ, Gomella LG. Ultrasonography and biopsy of the prostate. In: Wein AJ, editor. Campbell-Walsh Urology. 10th ed; 2011. p. 2735.

6. Hossack T, Patel MI, Huo A, Brenner P, Yuen C, Spernat D, Mathews J, Haynes A-M, Sutherland R, Del Prado W, et al. Location and pathological characteristics of cancers in radical prostatectomy specimens identified by Transperineal biopsy compared to Transrectal biopsy. J Urol. 2012;188(3):781-5.

7. Mazaheri Y, Shukla-Dave A, Muellner A, Hricak H. MR imaging of the prostate in clinical practice. Magn Reson Mater Phys Biol Med. 2008;21(6):379-92.

8. Hamoen E, de Rooij M, Witjes J, Barentsz J, Rovers M. Use of the prostate imaging reporting and data system (PI-RADS) for prostate Cancer detection with multiparametric magnetic resonance imaging: a diagnostic meta-analysis. Eur Urol. 2015;67(6):1112-21.

9. Siddiqui MM, Rais-Bahrami S, Hong T, Stamatakis L, Vourganti S, Nix J, Hoang AN, Walton-Diaz A, Shuch B, Weintraub M, et al. Magnetic resonance imaging/ultrasound-fusion biopsy significantly upgrades prostate Cancer versus systematic 12-core Transrectal ultrasound biopsy. Eur Urol. 2013;64(5):713-9.

10. Overduin CG, Futterer JJ, Barentsz JO. MRI-quided biopsy for prostate Cancer detection: a systematic review of current clinical results. Curr Urol Rep. 2013;14(3):209-13.

11. Park BK, Park JW, Park SY, Kim CK, Lee HM, Jeon SS, Seo SI, Jeong BC, Choi HY. Prospective evaluation of 3-T MRI performed before initial Transrectal ultrasound-guided prostate biopsy in patients with high prostate-specific antigen and no previous biopsy. Am J Roentgenol. 2011;197(5):W876-81.

12. Haffner J, Lemaitre L, Puech P, Haber G-P, Leroy X, Jones JS, Villers A. Role of magnetic resonance imaging before initial biopsy: comparison of magnetic resonance imaging-targeted and systematic biopsy for significant prostate cancer detection. BJU Int. 2011:108(8B):E171-8.

13. Quentin M, Blondin D, Arsov C, Schimmoeller L, Hiester A, Godehardt E, Albers $P$, Antoch $G$, Rabenalt R. Prospective evaluation of magnetic resonance imaging guided in-bore prostate biopsy versus systematic Transrectal ultrasound guided prostate biopsy in biopsy naive men with elevated prostate specific antigen. J Urol. 2014;192(5):1374-9.

14. Delongchamps NB, Peyromaure M, Schull A, Beuvon F, Bouazza N, Flam T, Zerbib M, Muradyan N, Legman P, Cornud F. Prebiopsy magnetic resonance imaging and prostate Cancer detection: comparison of random and targeted biopsies. J Urol. 2013;189(2):493-9.

15. Tonttila PP, Lantto J, Paakko E, Piippo U, Kauppila S, Lammentausta E, Ohtonen P, Vaarala MH. Prebiopsy multiparametric magnetic resonance imaging for prostate cancer diagnosis in biopsy-naive men with suspected prostate cancer based on elevated prostate-specific antigen values: results from a randomized prospective blinded controlled trial. Eur Urol. 2016;69(3):419-25.

16. Baco E, Rud E, Eri LM, Moen G, Vlatkovic L, Svindland A, Eggesbo HB, Ukimura $\mathrm{O}$. A randomized controlled trial to assess and compare the outcomes of two-core prostate biopsy guided by fused magnetic resonance and Transrectal ultrasound images and traditional 12-core systematic biopsy. Eur Urol. 2016;69(1):149-56.

17. Barentsz JO, Richenberg J, Clements R, Choyke P, Verma S, Villeirs G, Rouviere O, Logager V, Futterer JJ. ESUR prostate MR guidelines 2012. Eur Radiol. 2012;22(4):746-57.
18. Weinreb JC, Barentsz JO, Choyke PL, Cornud F, Haider MA, Macura KJ, Margolis D, Schnall MD, Shtern F, Tempany CM, et al. PI-RADS prostate imaging - reporting and data system: 2015, version 2. Eur Urol. 2016:69(1):16-40.

19. Kasivisvanathan V, Dufour R, Moore CM, Ahmed HU, Abd-Alazeez M, Charman SC, Freeman A, Allen C, Kirkham A, van der Meulen J, et al. Transperineal magnetic resonance image targeted prostate biopsy versus Transperineal template prostate biopsy in the detection of clinically significant prostate Cancer. J Urol. 2013;189(3):860-6.

20. Symons JL, Huo A, Yuen CL, Haynes A-M, Matthews J, Sutherland RL, Brenner P, Stricker PD. Outcomes of transperineal template-guided prostate biopsy in 409 patients. BJU Int. 2013;112(5):585-93.

21. Washino S, Okochi T, Saito K, Konishi T, Hirai M, Kobayashi Y, Miyagawa T. Combination of PI-RADS score and PSA density predicts biopsy outcome in biopsy naïve patients. BJU Int. 2017;119(2):225-33.

22. Miyagawa T, Ishikawa S, Kimura T, Suetomi T, Tsutsumi M, Irie T, Kondoh M, Mitake T. Real-time virtual sonography for navigation during targeted prostate biopsy using magnetic resonance imaging data. Int J Urol. 2010;17(10):855-60.

23. Pokorny MR, De Rooij M, Duncan E, Schroeder FH, Parkinson R, Barentsz JO, Thompson LC. Prospective study of diagnostic accuracy comparing prostate Cancer detection by Transrectal ultrasound-guided biopsy versus magnetic resonance (MR) imaging with subsequent MR-guided biopsy in men without previous prostate biopsies. Eur Urol. 2014;66(1):22-9.

24. Cool DW, Zhang X, Romagnoli C, Izawa JI, Romano WM, Fenster A. Evaluation of MRI-TRUS fusion versus cognitive registration accuracy for MRI-targeted, TRUS-guided prostate biopsy. Am J Roentgenol. 2015;204(1):83-91.

25. Kuru TH, Roethke MC, Rieker P, Roth W, Fenchel M, Hohenfellner M, Schlemmer H-P, Hadaschik BA. Histology core-specific evaluation of the European Society of Urogenital Radiology (ESUR) standardised scoring system of multiparametric magnetic resonance imaging (mpMRI) of the prostate. BJU Int. 2013;112(8):1080-7.

26. Grey ADR, Chana MS, Popert R, Wolfe K, Liyanage SH, Acher PL. Diagnostic accuracy of magnetic resonance imaging (MRI) prostate imaging reporting and data system (PI-RADS) scoring in a transperineal prostate biopsy setting. BJU Int. 2015;115(5):728-35.

\section{Ready to submit your research? Choose BMC and benefit from:}

- fast, convenient online submission

- thorough peer review by experienced researchers in your field

- rapid publication on acceptance

- support for research data, including large and complex data types

- gold Open Access which fosters wider collaboration and increased citations

- maximum visibility for your research: over $100 \mathrm{M}$ website views per year

At BMC, research is always in progress.

Learn more biomedcentral.com/submissions 